\title{
Prevention of Staphylococcus aureus biofilm formation on me- tallic surgical implants via controlled release of gentamicin
}

\author{
David J. McMillan ${ }^{1 \#}$, Cameron Lutton ${ }^{2 \#}$, Natalie Rosenzweig ${ }^{1,2}$, Kadaba S. Sriprakash ${ }^{1}$, Ben Goss ${ }^{2}$, \\ Michaela Stemberger ${ }^{2}$, Michael A. Schuetz ${ }^{2,3}$, Roland Steck ${ }^{2}$

\footnotetext{
${ }^{1}$ Bacterial Pathogenesis Laboratory, The Queensland Institute of Medical Research, Brisbane, Australia;

${ }^{2}$ Institute of Health and Biomedical Innovation, Queensland University of Technology, Brisbane, Australia;

${ }^{3}$ Trauma Services, Dept of Orthopaedics, Princess Alexandra Hospital, Woolloongabba, Australia.

Email: r.steck@qut.edu.au
}

Received 24 May 2011; revised 18 June 2011; accepted 4 July 2011.

\begin{abstract}
Implant associated infections are a critical health concern following orthopaedic surgery. Sustained local delivery of antibiotics has been suggested as a means of preventing these infections. Poly(D,L-lactide) (PDLLA) is a biodegradable polymer that has been used to coat implants for the delivery of antibiotics and other bioactive molecules. While effective, these studies show that antibiotics are released in a burst profile. Here we evaluated a method for controlled release of gentamicin from implant surfaces using the palmitate alkyl salt to decrease its solubility in aqueous solution. Steel Kirschner wires (K-wires) were coated with Gentamicin-palmitate (GP)-PDLLA, gentamicin sulphate (GS)-PDLLA or vancomycin sulphate (VS)-PDLLA, and elution of antibiotics from coated K-wires investigated using HPLC/MS/ MS. In contrast to burst antibiotic release from the GS-PDLLA and VS-PDLLA groups, GP was released in a slower sustained manner. Colonisation and initial attachment of Staphylococcus aureus Xen29 to gentamicin-coated $\mathrm{K}$-wires was reduced by $90 \%$ when compared to the non-coated control group. However there was no statistical difference in recovery of bacteria from GS or GP groups. Bacteria recovered from VS-PDLLA coated K-wires decreased by $36 \%$. Bioluminescence emitted by $S$. aureus Xen29 was also reduced over seven days in the antibiotic control groups, demonstrating that growth and biofilm development over the longer term was impaired by antibiotic-PDLLA coating. These results indicate that using alkyl salts of antibiotics may be an effective strategy for controlling the release of antibiotics from implants.
\end{abstract}

\footnotetext{
${ }^{\#}$ These two authors contributed equally to this work.
}

Keywords: Implant Infections; Poly(D,L-lactide); Biofilm; Gentamicin; Palmitate

\section{INTRODUCTION}

Medical implants and devices are a vital part of modern medical practice, including orthopaedics and trauma care, with an estimated 600,000 joint prostheses and two million fracture fixation devices used each year in the United States alone [1]. While improving health outcomes for patients, implant associated infections have become a leading cause of nosocomial infection. Between $2 \%$ of elective orthopaedic surgeries [2], and up to $44 \%$ of individuals with open fractures develop postoperative infections [3]. Treatment of these infections includes long term antibiotic therapy, possible removal of the device and/or debridement of surrounding tissue $[4,5]$. In the most severe cases, amputation may be required. In addition to the increased health burden, treatment of implant associated infections poses a significant economic cost to the health sector [6].

Treatment of open fracture associated infections has added complications. The initial trauma exposes normally sterile body sites to the external environment and increases the chance of infection. The presence of necrotic anaerobic tissue also promotes infection, and damage to vasculature around the wound site can prevent systemically administered antibiotics from reaching the site of infection [7]. Osteomyelitis is another significant complication associated with open fractures, and delivery of antibiotics at concentrations required to kill bacteria in the intramedullary cavity is difficult to establish and maintain. Staphylococcus aureus and coagulase negative staphylococci are the major bacterial pathogens associated with osteomyelitis [8-11]

Current approaches towards reduction of implant related infection include inhibition of initial bacterial ad- 
hesion, coating implants with polymers which have inherent antibacterial activity or by loading of polymers with antimicrobial agents [12-16]. Polymer-antibiotic coatings are a particularly attractive option for treatment of open fractures as these coatings will prevent initial bacterial attachment and colonisation of the implant, and release of antibiotics from the implant surface may also eradicate bacteria from the surrounding tissues. Poly-D, L-lactide (PDLLA) is a biodegradable non-toxic polymer that has been used to coat implants with antibiotics and other bioactive molecules [17,18]. PDLLA-gentamicin sulphate combinations have previously been shown to reduce implant colonisation by $S$. epidermidis in in vitro and in vivo models [15]. However, immiscibility between the antibiotic salt and the polymer may lead to phase separation during coating, with sustained release profiles being difficult to achieve. Previous studies have reported a rapid burst release of gentamicin sulphate from the surface of implants in the first hour after immersion in PBS [15]. Using poorly water soluble salts of antibiotics has been suggested as a method to improve miscibility during coating and their temporal prophylactic efficiency [19]. In the present study we compared the ability of a PDLLA/gentamicin palmitate coating to provide sustained release and inhibit $S$. aureus colonisation and biofilm formation on the surface of steel Kirschner wires, and compared its performance with PDLLA coatings containing gentamicin sulphate and vancomycin sulphate.

\section{MATERIALS AND METHODS}

\subsection{Bacterial Strains and Media}

Staphylococcus aureus Xen29 is a derivative of the biofilm forming $S$. aureus 12600 that has been recombinantly altered by the introduction of a lux operon derived from Photorhabdus luminescens [20], resulting in expression of luciferase. Unless otherwise indicated $S$. aureus Xen29 was grown in Tryptic Soy Broth (TSB), Tryptic Soy Agar (TSA) or Columbia agar at $37^{\circ} \mathrm{C}$. The minimum inhibitory concentrations (MIC) of gentamicin and vancomycin for $S$. aureus Xen 29 are reported as $12.5 \mathrm{mg} \cdot \mathrm{L}^{-1}$ and $0.78 \mathrm{mg} \cdot \mathrm{L}^{-1}$ respectively [21]. The MIC for gentamicin palmitate was determined to be 25 $\mathrm{mg} \cdot \mathrm{L}^{-1}$.

\subsection{PDLLA-Antibiotic Coating of Kirschner Wires}

Three antibiotic-PDLLA combinations, gentamicin sulphate (GS), gentamicin palmitate (GP) and vancomycin sulphate (VS) were used in this study. The PDLLA used (Resomer R203) was a racemic mixture of the D- and L-enantiomers of lactic acid with a molecular weight of $29 \mathrm{kDa}$. Polymer-antibiotic solutions were prepared with the aim of maximising the antibiotic concentration whilst simultaneously maintaining solubility of all components. Gentamicin sulphate (Sigma-Aldrich AG Pty. Ltd., Castle Hill, Australia) was first dissolved in a 50:50 mixture of $\mathrm{dH}_{2} \mathrm{O}$ and PEG 1000 (Sigma-Aldrich) to improve miscibility with the polymer at a concentration of $22 \mathrm{mg} \cdot \mathrm{ml}^{-1}$. PDLLA was dissolved in DMSO to a final concentration of $62.5 \mathrm{mg} \cdot \mathrm{ml}^{-1}$. The gentamicin sulphate solution and PDLLA solutions were mixed in a $1: 10$ ratio with stirring at $50^{\circ} \mathrm{C}$ to produce a clear solution. The PDDLA-gentamicin palmitate (pentakis alkyl salt) solution was prepared in a similar fashion. However the GP was first dissolved in chloroform, rather than $\mathrm{dH}_{2} \mathrm{O}$ at a concentration of $29 \mathrm{mg} \cdot \mathrm{ml}^{-1}$. The PDDLA-vancomycin solution was prepared by dissolving vancomycin $\left(22 \mathrm{mg} \cdot \mathrm{ml}^{-1}\right)$ and PDLLA (133.3 $\mathrm{mg} \cdot \mathrm{ml}^{-1}$ ) in DMSO. Steel Kirschner wires (K-wires, 1.8 $\mathrm{mm}$ diameter) were manufactured by Synthes (Switzerland). The K-wires were cut to a length of approximately $30 \mathrm{~mm}$ and dipcoated in $5 \mathrm{ml}$ of coating solution. The wire was removed and dried in a vacuum oven for $24 \mathrm{hr}$ at $50^{\circ} \mathrm{C}$, and then the coating process repeated to increase antibiotic content on each $\mathrm{K}$-wire. Each wire was inspected visually after coating for completeness of the coating. Coated wires were promptly used for all experiments.

\subsection{Abrasion Test}

$\mathrm{K}$-wires were cut to $30 \mathrm{~mm}$ length and weighed prior to, and after coating. The K-wires were then inserted and passed through $1.8 \mathrm{~mm}$ diameter drill holes in $25 \mathrm{~mm}$ thick bovine cortical bone specimens and reweighed. After gently washing in methanol to remove dislodged polymer and bone, the samples were weighed a fourth time. The total and percentage loss of coating mass for each K-wire was then calculated.

\subsection{Elution Assays}

To determine the rate of release of antibiotics from implant surface, coated K-wires, cut into three $50 \mathrm{~mm}$ long segments, were placed in $10 \mathrm{ml}$ tubes containing $10 \mathrm{ml}$ of PBS (three segments per tube). The samples were incubated at $37^{\circ} \mathrm{C}$ and $500 \mu \mathrm{l}$ aliquots collected at $0 \mathrm{~h}$, $10 \mathrm{~min}, 1 \mathrm{~h}, 2 \mathrm{~h}, 3 \mathrm{~h}, 4 \mathrm{~h}, 8 \mathrm{~h}, 24 \mathrm{~h}$, and $48 \mathrm{~h}$ and 7 days. Antibiotic concentrations in the samples were determined by HPLC/MS/MS using an AB/Sciex API4000Q (AB/Sciex Concord, Ontario, Canada) mass spectrometer equipped with an electrospray (TurboV) interface coupled to a Shimadzu Prominence HPLC system (Shimadzu Corp., Kyoto, Japan), resulting in a detection limit of $0.5 \mathrm{mg} \cdot \mathrm{L}^{-1}$.

\subsection{Bacterial Attachment Assays}

To measure attachment to K-wires, S. aureus Xen29 was 
grown overnight in TSB. After washing, the bacteria were diluted in PBS to $\mathrm{OD}_{595}$ of 0.1 . This suspension was further diluted 1:10 in PBS. A $1.3 \mathrm{ml}$ aliquot of this suspension, containing approximately $10^{6} \mathrm{CFU}$ of $S$. aureus was then added to $1.5 \mathrm{ml}$ microfuge tubes, and individual $\mathrm{K}$-wires added to each tube. After incubation at $37^{\circ} \mathrm{C}$ for two hours, the $\mathrm{K}$-wires were recovered, washed three times in PBS to remove residual antibiotic and transiently attached bacteria, and placed in $1.3 \mathrm{ml}$ of a $0.5 \%$ trypsin/PBS solution at room temperature. Bacteria were subsequently dislodged from the surface by sonication at $47 \mathrm{KHz}$ for $15 \mathrm{~min}$ at room temperature. The suspension was then centrifuged to concentrated bacteria, and resuspended in PBS. After serial dilution of the resuspended samples, aliquots were plated onto TSB. The percentage of CFU recovered from PDLLA-antibiotic groups when compared to the average CFU recovered from the uncoated and PDLLA control groups was determined for each set of experiments.

\subsection{Inhibition of Biofilm Growth}

Growth of $S$. aureus Xen29 biofilms on the surface of K-wires was monitored for 7 days using an IVIS ${ }^{\circledR} 100$ Series (Caliper Life Sciences, Hopkinton, USA) camera [20]. Coated and uncoated K-wires were placed in 6-well tissue culture plate. Five millilitres of TSB containing approximately $10^{5} \mathrm{CFU}$ of $S$. aureus Xen29 was then added to each well, and the plate incubated overnight without shaking. Next day, the K-wires were recovered, gently rinsed, and transferred to a new 6-well plate containing fresh TSA. Bioluminescence was captured using the IVIS camera approximately $20 \mathrm{~min}$ after transfer to the new 6-well plate. The bioluminescence in a defined region surrounding each $\mathrm{K}$-wire was then quantified using Living image software. After visualisation, the K-wires were placed in a new 6-well plate, and fresh media (without bacteria) added, and incubation continued.

\subsection{Statistical Analysis}

T-tests were used to assess the statistical significance between mean values of experimental and control groups in the study. Differences were considered significant at $p \leq$ 0.05 .

\section{RESULTS}

\subsection{Loading and Mechanical Strength}

The average mass of the GS, GP and VS coating on the $\mathrm{K}$-wires was $5.1 \mathrm{mg}, 3.2 \mathrm{mg}$ and $3.0 \mathrm{mg}$ respectively. To determine the mechanical robustness of the coatings in the face of abrasive forces similar to that expected in orthopaedic surgery, the K-wires were passed through $1.8 \mathrm{~mm}$ drill holes in bovine cortical bone, and re- weighed (Figure 1).

The GS group lost an average $1.9 \mathrm{mg}$ (37\% of coating mass) per K-wire, whereas the GP group lost an average $0.5 \mathrm{mg}(16 \%)$. The difference in total loss and percentage loss for the two groups was not statistically significant. Very little loss of mass $(0.06 \mathrm{mg}, 1.5 \%)$ was observed in the VS group.

\subsection{Antibiotic Release}

Antibiotics coated to the surface of implants have two functions. Firstly, antibiotic on the implant should prevent colonisation by viable bacteria, and subsequent biofilm development. Secondly, released antibiotic should kill bacteria in the immediate vicinity of the implant. The release of antibiotic from the three coating groups was investigated by immersing coated K-wires in PBS, and measuring concentrations of eluates over time (Figure 2). For the GS coated K-wires, the majority (71\%) of the gentamicin was rapidly released in the first $10 \mathrm{~min}$. after immersion and reached a maximum concentration at 2 hours. The release of vancomycin was even more rapid with it reaching $91 \%$ of its maximum solution concentration within the first $10 \mathrm{~min}$. after immersion. Like the GS group, the maximum concentration of vancomycin in the solution was reached after two hours. In contrast to the burst release observed in these two groups, the GP group had a much slower rate of release. At 10 min., the mean concentration of gentamicin in the eluate of this group was $6.5 \mathrm{mg} \cdot \mathrm{L}^{-1} \pm 2.3 \mathrm{mg} \cdot \mathrm{L}^{-1}$, seven fold lower than measured in the GS group and 8 fold lower than measured in VS group at the same time point. The GP group continued to provide a sustained release for up to $48 \mathrm{hrs}$ and peaked at a concentration of $24 \pm 5 \mathrm{mg} \cdot \mathrm{L}^{-1}$, 2.5 times less than either the GS or VS groups.

\subsection{Inhibition of Colonisation of K-Wires}

To test the ability of antibiotic-PDLLA to inhibit $S$. aureus colonisation, K-wires were incubated in static $S$. aureus suspension, and then washed to remove residual antibiotic. Adhered bacteria were then dislodged by
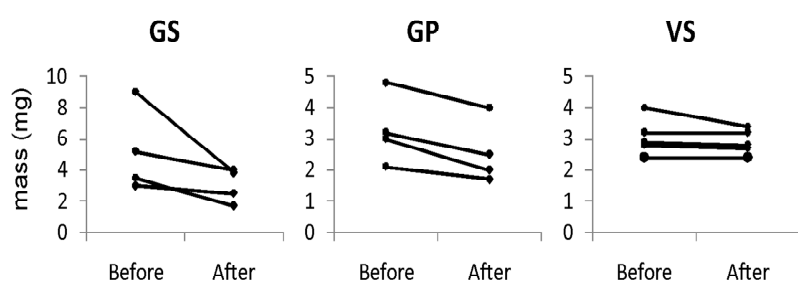

Figure 1. Loss of coating mass after abrasion testing. Coated $\mathrm{K}$-wires were weighed prior to, and after being passed through a $1.8 \mathrm{~mm}$ bore hole in bovine cortical bone. Data points representing individual $\mathrm{K}$-wires prior to and after passing through the bone are connected by the lines. 
sonication, serially diluted and plated onto Columbia agar. The total and percentage CFU in the experimental group, relative to the CFU recovered from control groups (uncoated and PDLLA groups) was then determined for each individual experiment (Figure 3). Over

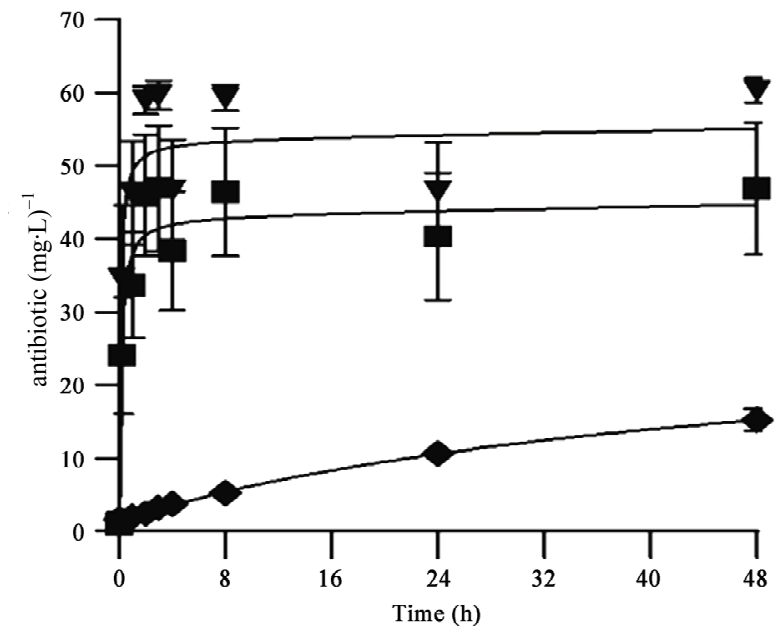

Figure 2. Elution of antibiotic from gentamicin sulphate $(\boldsymbol{\square}) \mathrm{S}$ $(n=8)$, gentamicin palmitate $(\diamond)(n=5)$ and vancomycin $(\boldsymbol{\nabla})$ coated K-wires. K-wires were immersed in PBS, and $500 \mu \mathrm{l}$ aliquots taken over 48 hours. Data is presented as the mean concentration of antibiotic in the gentamicin sulphate $(n=8)$ and gentamicin palmitate $(n=5)$ and vancomycin $(n=8)$ solutions. Error bars represent the SEM.

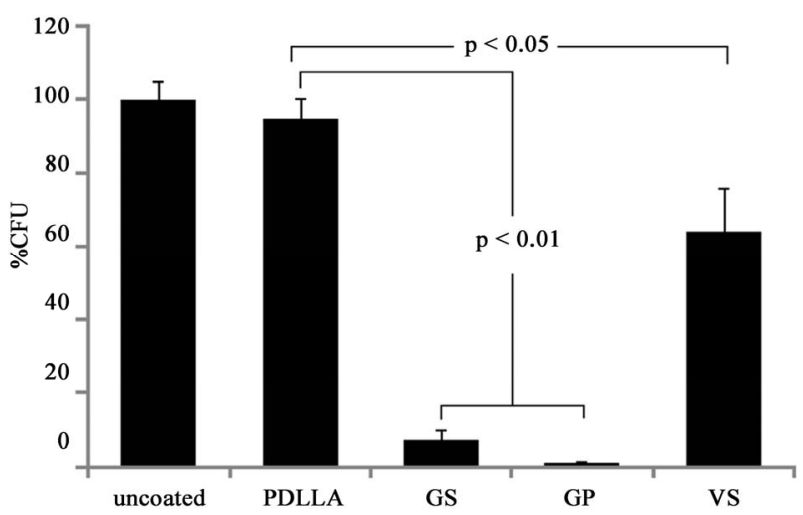

Figure 3. PDLLA-antibiotic coating inhibit bacterial colonisation of K-wires. K-wires were incubated with a suspension of $S$. aureus Xen 29 for two hours. Bacteria were dislodged from the $\mathrm{K}$-wire by incubation with $5 \%$ trypsin and sonication. The suspensions were serially diluted and plated on agar, incubated overnight and CFU determined. Data is presented as the mean percentage CFU remaining on each antibiotic-PDLLA group of $\mathrm{K}$-wires when compared to the average CFU present on the "uncoated" and "PDLLA" coated control K-wires. All experiments were performed at least in triplicate, with at least three $\mathrm{K}$-wires represented in each independent experiment. Error bars represent SEM. Differences between the mean \%CFU of control and experimental groups were found to be statistically significant. all, significantly lower bacterial numbers were recovered from the antibiotic coated K-wires. The mean bacterial CFU recovered from uncoated and PDLLA coated $\mathrm{K}$-wires was $1.2 \times 10^{5} \pm 5.8 \times 10^{4} \mathrm{CFU}$ and $1.0 \times 10^{5} \pm$ $5.8 \times 10^{4} \mathrm{CFU}$ respectively. The mean CFU recovery of bacteria from the GS $\left(1.1 \times 10^{4} \pm 6.9 \times 10^{3}\right)$, GP $(1.0 \times$ $\left.10^{3} \pm 6.3 \times 10^{2}\right)$ and VS groups $\left(8.1 \times 10^{4} \pm 4.2 \times 10^{4}\right)$ was significantly lower. There was a $93 \%$ reduction in CFU on GS and $99 \%$ reduction of CFU on GP coated wires. The $36 \%$ reduction in colonisation observed in the VS group was not as pronounced but still represents a significant decrease in comparison to the control group $(p<0.05)$.

\subsection{Inhibition of Biofilm Formation}

The results of the colonisation studies demonstrated that all the antibiotic coatings inhibited initial colonisation events. To assess whether the reduced colonisation had any impact on biofilm formation, we next incubated Kwires with recombinant $S$. aureus Xen29 over a period of seven days, and captured bioluminescence emitted by the bacteria using an IVIS CCD camera (Figure 4). The advantage of the IVIS system over other methods for bacterial monitoring lies in the ability to measure the same sample at multiple time points. For the uncoated group, an initial signal intensity of $3.1 \times 10^{4}$ photon/s was recorded on day 1 . The luminescence then increased to a maximum on the fourth day of the incubation, and declined slowly after this time point. In contrast to other groups, the photonic signal observed on day 1 for the PDLLA control group exceeded $10^{5}$ photons/s. The relatively higher level of colonisation, as measured by luminescence, was maintained throughout the experiment. After this time point, biofilm growth continued to increase over the course of the experiment. For the antibi-

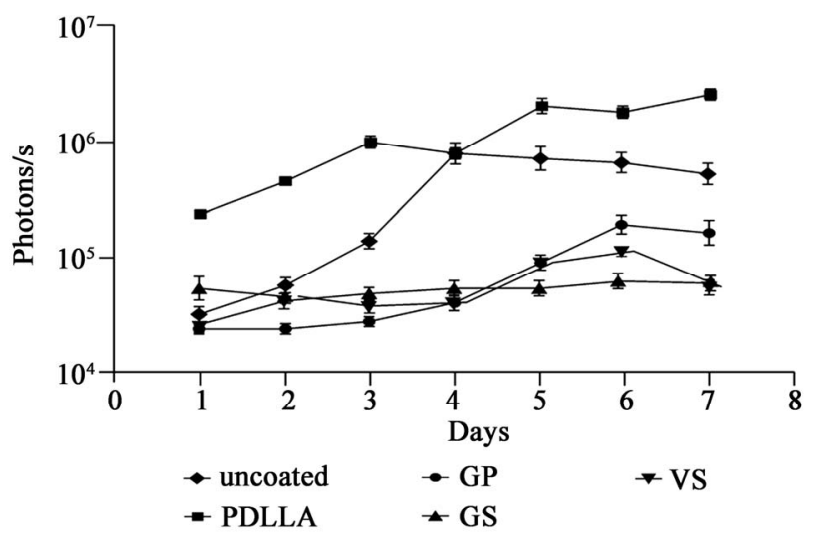

Figure 4. Biofilm formation on the surface of K-wires. Kwires were incubated with $S$. aureus Xen29 overnight and bioluminescence emitted by the bacteria monitored daily using an IVIS CCD camera. Figure shows mean luminescent signal intensity of control and experimental groups measured over the seven days of the experiment. 
otic-PDLLA groups, the signal intensity observed on the first day was similar to the uncoated sample.

For the GS group, signal intensity rose to a maximum of $6.5 \times 10^{4}$ photon/s on day 6 . However this was not significantly greater than the intensity on day 1 . Similarly, no statistical increase in the intensity of signal in the vancomycin group was observed. The GP group also showed no increase in intensity over the first four days. While a small increase was observed in the last two days of the experiment, the signal intensity was still ten times lower than the PDDLA group on comparable days. Using the IVIS camera, we were also able to detect bacteria in the culture supernatant for each sample for each day of the experiment (data not shown). Taken together these results indicated that the three antibiotic groups were effective at reducing biofilm formation on the K-wires.

\section{DISCUSSION}

Infections are still among the leading causes for complications in orthopaedic surgery, ranging in frequency from $1 \%-5 \%$ in elective, joint replacement surgery, to higher rates in osteosynthesis of open fractures [9]. They are painful, difficult to treat and can lead to implantrelated osteomyelitis. Furthermore, these complications cause prolonged hospitalisation and are associated with significantly increased costs. Therefore, antibiotic prophylaxis, usually via intravenous administration, is a routine procedure in these surgeries [22]. Although this systemic prophylaxis is successful in most cases, delivery to wounds can be problematic due to the presence of ischemic tissue. The difficulty in delivering antibiotics to these tissues also increases the risk of the growth of implant associated biofilms, which show high resistance to antibiotics and the immune system and often require implant removal [23,24].

The development of biodegradable implant coating techniques that provide both local delivery of antibiotics and prevent biofilm formation is an attractive approach for the prevention of these infections. Local delivery ensures that high concentrations of antibiotic are delivered at the wound site where infections are most likely to occur, whilst antibiotics on the surface of the implant may prevent bacterial colonisation. Antibiotic release from these coatings occurs in a burst release fashion, which is desirable in some clinical situations (e.g. open fractures with contamination), but which on its own may not be ideal to prevent infections caused by delayed or repeated exposure to bacteria. To that end we investigated the use of an alkyl salt of gentamicin, gentamicin palmitate, to limit burst release and extend delivery time from PDLLA coated implants.

Antibiotic miscibility with the coating solution had a large effect on the film toughness. Increasing the misci- bility of the antibiotic with PDLLA resulted in tougher films with the gentamicin palmitate/PDLLA film as evident by its losing less mass when compared to the gentamicin sulphate/PDLLA film (although the difference was not statistically significant). Vancomycin, which was miscible in all proportions with PDLLA, lost very little mass under our test conditions. We attribute the difference in mass loss to the disruption that immiscible particles, such as gentamicin sulphate, cause to the film structure upon drying. It is well known that the interface between immiscible particles and polymer films has a great influence on the toughness of the films [25,26]. Particles with no adhesive interaction with the polymer essentially act as porogens, creating defects in the film. Conversely, the miscibility of vancomycin provided a more even distribution and no reduction in film toughness. Whilst not specifically reported here, it was possible to include too much vancomycin in the films with concentrations over $50 \mathrm{mg} \cdot \mathrm{ml}^{-1}$ completely disrupting stable film formation. These coatings were shed from the implant surface (data not shown).

The release rates of the gentamicin and vancomycin in our study are similar to those reported when no coating was used on implants [27]. Under our time frame the polymer did not degrade significantly or show any effect of controlling the release as has been reported by other authors [28]. We attribute this difference to our coating procedure. Our procedure produced very smooth films unlike those prepared by Aviv et al. whose coating procedure produced slightly porous films with phase separated domains of antibiotic. In their system early prolonged release is most likely due to an increased path length of antibiotic through pores in the polymer surface, not degradation mediated release as they saw at later time points. It is likely that our procedure of improving the solution miscibility of the antibiotics with the polymer and the smoothness of the films that were created reduced the porosity and effectively trapped any nonsurface bound antibiotic in the polymer. Our release results, coupled with the long degradation time of PDLLA $[29,30]$, suggest that the release of these antibiotics over the time frame considered is mostly due to residual antibiotic on the surface. We demonstrated that the use of gentamicin palmitate does result in an extended delivery compared to both gentamicin sulphate and vancomycin, but in less total antibiotic release. We would expect that the longer release to be due to a decreased solubility of the palmitate salt in PBS. Similar to gentamicin sulphate and vancomycin, this would be surface adsorbed gentamicin palmitate that slowly dissolves in PBS. There are two possible explanations for a lower total amount of gentamicin palmitate released. As the salt is sparingly soluble in PBS it may be that the solubility limit was 
reached in the volume of PBS used in the elution experiment. Whilst we cannot totally rule this explanation out, we believe that the low total amount released (24 $\left.\mathrm{mg} \cdot \mathrm{L}^{-1}\right)$ is well below the solubility limit of the antibiotic in PBS. The media being changed constantly in the biofilm experiments and the increase in bacteria at four days also suggests that the elution experiments closely reflect the amount of antibiotic able to be released. A more likely explanation is that our coating procedure results in less total antibiotic adsorbed to the surface. Care was taken to ensure that similar molar concentrations of gentamicin were present in both the sulphate and palmitate coating solutions and that the coating procedures were as similar as possible. However the differing solvents used, i.e. $\mathrm{H}_{2}$ 0/PEG1000/DMSO combination for the gentamicin sulphate and just chloroform for the gentamicin palmitate likely resulted in differing levels of partitioning of the antibiotic to the surface during the coating procedure. It should be possible to increase the amount of gentamicin palmitate on the surface simply by increasing the coating solution concentration or increasing the number of dip coatings. This would certainly increase the total amount of antibiotic released and may possibly extend the delivery time. The slower sustained release of antibiotic may be particularly effective in combating late onset osteomyelitis [31].

Despite having different delivery profiles all three antibiotics reduced the numbers of attached bacteria. Both salts of gentamicin were equally effective in reducing bacterial attachment and were in fact better than vancomycin at the two hour time point used for testing. Given that all antibiotics were present in concentrations above their minimum inhibitory concentrations this difference may be due to vancomycin's slow bactericidal activity compared with gentamicin [32]. The longer incubation times in the biofilm assays, and similar reduction in bacterial load on gentamicin and vancomycin-coated $\mathrm{K}$-wires would seem to support this. Unlike the results of Gollwitzer et al. [13] we did not see a reduction in the attachment of viable bacteria on PDLLA-coated steel when compared to uncoated steel. The contradicting results may be due to the different bacterial species used, (S. aureus vs S. epidermidis) although PDLLA coated wires have shown no inhibitory effect on bacterial growth in vivo [33].

Under the conditions used in the study, the presence of antibiotics on the K-wires was able to reduce biofilm formation for at least seven days, as determined through measurement of bioluminescence. Although vancomycin was less effective in preventing initial colonisation, it is as effective as the gentamicin salts in retarding biofilm formation compared with both the uncoated and PDLLA coated wires. Our results also showed that an increase in bacterial load after day 4 occurred for K-wires coated with gentamicin palmitate, but not gentamicin sulphate. This suggests that some viable $S$. aureus, below the detection limits of the assay, remained on the GP coated $\mathrm{K}$-wires on days 1 - 4. The sustained elution profile of the GP group, resulting in reduced concentrations of gentamicin at specific time-points, may have enabled small numbers of bacteria to survive. The subsequent increase in bacterial numbers after four days suggests that the majority of gentamicin had been released by this time, allowing the growth of $S$. aureus. This contrasts with the high concentrations of gentamicin in solution associated with the burst kinetics of the gentamicin sulphate group, possibly killing all bacteria in the assay at an early time-point. These observations highlight the importance of achieving the appropriate antibiotic elution kinetics in the clinical environment. While it is desirable for antibiotic to elute from implants in appropriate concentrations to kill surrounding bacteria, sufficient antibiotic must also remain attached to the implants to help prevent later colonisation events. One of the drawbacks of this protocol is that we did not challenge the coated rods after the burst release was complete, as might be expected in vivo.

Collectively these results show that the presence of antibiotics on implants can reduce bacterial attachment and biofilm formation and that antibiotic/polymer miscibility affects the coating toughness, but not the delivery profile. We have demonstrated that gentamicin-palmitate has a reduced rate of release from metal surgical implants, but that this did not translate to a significant reduction in bacterial attachment and biofilm formation in our model. Ultimately, the effect of different release profiles on the prevention of implant-related bone infections will have to be tested and demonstrated in suitable pre-clinical in vivo animal models. Nevertheless the sustained release kinetics of gentamicin palmitate from PDLLA, as demonstrated in our in vitro experiments, may represent a strategy for improving the release of antibiotics from polymer coatings for combating infection.

\section{ACKNOWLEDGEMENTS}

This work was supported by a grant from the Wesley Research Institute (2007/04), Brisbane, Australia.

The authors would like to thank Prof Axel Stemberger from the Technical University, Munich (Germany) for his assistance with the conceptual design for this study, and Dr. Geoff Eaglesham from Queensland Health Scientific Services for assistance with elution assays.

\section{REFERENCES}

[1] Darouiche, R.O. (2001) Device-associated infections: A macroproblem that starts with microadherence. Clinical Infectious Diseases, 33, 1567-1572. doi:10.1086/323130 
[2] Darouiche, R.O. (2004) Treatment of infections associated with surgical implants. The New England Journal of Medicine, 350, 1422-1429. doi:10.1056/NEJMra035415

[3] McGraw, J.M. and Lim, E.V. (1988) Treatment of open tibial-shaft fractures. External fixation and secondary intramedullary nailing. Journal of Bone and Joint Surgery, 70, 900-911.

[4] Campoccia, D., Montanaro, L. and Arciola, C.R. (2006) The significance of infection related to orthopedic devices and issues of antibiotic resistance. Biomaterials, 27, 2331-2339. doi:10.1016/j.biomaterials.2005.11.044

[5] Wiedel, J.D. (2002) Salvage of infected total knee fusion: The last option. Clinical Orthopaedics and Related Research, 404, 139-142. doi:10.1097/00003086-200211000-00024

[6] Sugarman, B. and Young, E.J. (1989) Infections associated with prosthetic devices: Magnitude of the problem. Infectious Disease Clinics of North America, 3, 187-198.

[7] Hughes, S.P. (1992) Antibiotic penetration into bone in relation to the immediate management of open fractures: A review. Acta Orthopaedica Belgica, 58, 217-221.

[8] Lew, D.P. and Waldvogel, F.A. (2004) Osteomyelitis. The Lancet, 364, 369-379.

doi:10.1016/S0140-6736(04)16727-5

[9] Harris, L.G. and Richards, R.G. (2006) Staphylococci and implant surfaces: A review. Injury, 37, S3-S14. doi:10.1016/j.injury.2006.04.003

[10] Wenzel, R.P. (1991) Epidemiology of hospital acquired infections. In: Balows, A. Jr., Hausler, W.J., Herman, K.L., Isenberg, H.D. and Shadomy, H.J., Eds., Manual of Clinical Microbiology, American Society for Microbiology, Washington, DC, 147-150.

[11] Dirschl, D.R. and Almekinders, L.C. (1993) Osteomyelitis. Common causes and treatment recommendations. Drugs, 45, 29-43. doi:10.2165/00003495-199345010-00004

[12] An, Y.H., Stuart, G.W., McDowell, S.J., McDaniel, S.E., Kang, Q., et al. (1996) Prevention of bacterial adherence to implant surfaces with a crosslinked albumin coating in vitro. Journal of Orthopaedic Research, 14, 846-849. doi:10.1002/jor.1100140526

[13] Gabriel, G.J., Maegerlein, J.A., Nelson, C.F., Dabkowski, J.M., Eren, T., et al. (2009) Comparison of facially amphiphilic versus segregated monomers in the design of antibacterial copolymers. Chemistry, 15, 433-439. doi:10.1002/chem.200801233

[14] Peltonen, L.I., Kinnari, T.J., Aarnisalo, A.A., Kuusela, P. and Jero, J. (2007) Comparison of bacterial adherence to polylactides, silicone, and titanium. Acta Otolaryngology, 127, 587-593. doi:10.1080/00016480600987792

[15] Gollwitzer, H., Ibrahim, K., Meyer, H., Mittelmeier, W., Busch, R., et al. (2003) Antibacterial poly(D,L-lactic acid) coating of medical implants using a biodegradable drug delivery technology. Journal of Antimicrobial Chemotherapy, 51, 585-591. doi:10.1093/jac/dkg105

[16] Kenawy, El-R., Worley, S.D. and Broughton, R. (2007) The chemistry and applications of anti-microbial polymers: A state-of-the-art review. Biomacromolecules, 8, 1359-1384. doi:10.1021/bm061150q

[17] Schmidmaier, G., Lucke, M., Wildemann, B., Haas, N.P. and Raschke, M. (2006) Prophylaxis and treatment of implant-related infections by antibiotic-coated implants: A review. Injury, 37, S105-S112.

doi:10.1016/j.injury.2006.04.016

[18] Schmidmaier, G., Wildemann, B., Bail, H., Lucke, M., Fuchs, T., et al. (2001) Local application of growth factors (insulin-like growth factor-1 and transforming growth factor-b1) from biodegradable poly(D,L-lactide) coating of osteosynthetic implants accelerates fracture healing in rats. Bone, 28, 341-350. doi:10.1016/S8756-3282(00)00456-7

[19] Vogt, S., Ku, K.-D., Ege, W., Pawlik, K., Schnabelrauch, M. (2003) Novel polylactide-based release systems for local antibiotic therapies. Materialwissenschaft und Werkstofftechnik, 34, 1041-1047. doi:10.1002/mawe. 200300701

[20] Kadurugamuwa, J.L., Sin, L., Albert, E., Yu, J., Francis, K., et al. (2003) Direct continuous method for monitoring biofilm infection in a mouse model. Infection and Immunity, 71, 882-890. doi:10.1128/IAI.71.2.882-890.2003

[21] Xiong, Y.Q., Willard, J., Kadurugamuwa, J.L., Yu, J., Francis, K.P., et al. (2005) Real-time in vivo bioluminescent imaging for evaluating the efficacy of antibiotics in a rat Staphylococcus aureus endocarditis model. Antimicrobial Agents and Chemotherapy, 49, 380-387. doi:10.1128/AAC.49.1.380-387.2005

[22] Periti, P., Mini, E. and Mosconi, G. (1998) Antimicrobial prophylaxis in orthopaedic surgery: The role of teicoplanin. Journal of Antimicrobial Chemotherapy, 41 , 329-340. doi:10.1093/jac/41.3.329

[23] Schmidmaier, G., Lucke, M., Wildemann, B., Haas, N.P. and Raschke, M. (2006) Prophylaxis and treatment of implant-related infections by antibiotic-coated implants: A review. Injury, 37, S105-S112. doi:10.1016/j.injury.2006.04.016

[24] Stewart, P.S. and Costerton, J.W. (2001) Anti-biotic resistance of bacteria in biofilms. The Lancet, 358, 135-138. doi:10.1016/S0140-6736(01)05321-1

[25] Felton, L.A., O'Donnell, P.B. and McGinity, J.W. (2008) Mechanical properties of polymeric films prepared from aqueous dispersions. Drugs and the Pharmaceutical Sciences, 176, 105-128.

[26] Hogan, J.E. (1995) Film-coating materials [for pharmaceuticals] and their properties. In: Cole, G., Ed., Pharmaceutical Coating Technology, Taylor and Francis, London, 6-52.

[27] Darouiche, R., Mansouri, M., Zakarevicz, D., AlSharif, A. and Landon, G. (2007) In Vivo Efficacy of antimicrobial-coated devices. Journal of Bone and Joint Surgery American, 89, 792-797. doi:10.2106/JBJS.F.00414

[28] Aviv, M., Berdicevsky, I. and Zilberman, M. (2007) Gentamicin-loaded bioresorbable films for prevention of bacterial infections associated with orthopedic implants. Journal of Biomedical Materials Research A, 83, 10-19. doi:10.1002/jbm.a.31184

[29] Suuronen, R., Pohjonen, T., Hietanen, J. and Lindqvist, C. (1998) A 5-year in vitro and in vivo study of the biodegradation of polylactide plates. Journal of Oral and Maxillofacial Surgery, 56, 604-614.

[30] Leenslag, J.W., Pennings, A.J., Bos, R.R., Rozema, F.R. and Boering, G. (1987) Resorbable materials of poly(Llactide). VII. In vivo and in vitro degradation. Biomate- 
rials, 8, 311-314. doi:10.1016/0142-9612(87)90121-9

[31] Gustilo, R.B., Gruninger, R.P. and Davis, T. (1987) Classification of type III (severe) open fractures relative to treatment and results. Orthopedics, 10, 1781-1788.

[32] Sakoulas, G., Moise-Broder, P.A., Schentag, J., Forrest, A., Moellering, R.C. Jr., et al. (2004) Relationship of MIC and bactericidal activity to efficacy of vancomycin for treatment of methicillin-resistant Staphylococcus au- reus bacteremia. Journal of Clinical Microbiology, 42, 2398-2402. doi:10.1128/JCM.42.6.2398-2402.2004

[33] Lucke, M., Wildemann, B., Sadoni, S., Surke, C., Schiller, R., et al. (2005) Systemic versus local application of gentamicin in prophylaxis of implant-related osteomyelitis in a rat model. Bone, 36, 770-778.

doi:10.1016/i.bone.2005.01.008 